\title{
Features Tagging Technique in the Sports Video Analysis
}

\author{
WU Yongwang ${ }^{1, \text { a }}$ \\ ${ }^{1}$ Jiangxi college of foreign study, NanChang, 330099, China \\ awuyongwang@yeah.net
}

Keywords: Features Tagging; Video Analysis; Sports Training

\begin{abstract}
With the rapid development of computer networks and video capture technology, resulting in a large number of sports video data, how to effectively and quickly retrieve the part of coaches or athletes need from these data, as a problem to be solved. Video marked its description given according to the video semantic content embodied, on this basis, can achieve efficient video retrieval. To be precise and reliable video capture sports, it is very difficult, and it is a task of great challenging. For the research in this field, both in theory or practical aspects are of great significance. It is based on this fact, the author under the teacher's instructions, for this cutting-edge research made some attempt to achieve a simple sports-based video capture system.
\end{abstract}

\section{Introduction}

In recent years, the motion capture technology in the field of computer animation frequently impact is growing, has aroused great concern of the majority of experts and scholars. However, the existing motion capture technology exists to capture most of the devices too expensive, performers and other shortcomings action is limited, thus restricting the further promotion and application of the technology. To solve this problem, this paper proposes a sports information extracted from the video, and use this to generate motion capture files [1-2]. This is a computer vision research a hot issue in recent years, the field of sports analysis, human-machine interfaces, virtual reality and animation, etc. have a wide range of application.

The sport caught on video to break the traditional limitations of motion capture, it does not require any extra auxiliary equipment, do not need the performer who bundled sensor allows performers free, arbitrary action display, thus ensuring the human action The free-flowing, and very low cost. In a sense, our study will be a simple, inexpensive motion capture system, it can be said to replace an existing motion capture system, it can be done by measurement of video in sports stores and reproduction. In this paper, we combine motion capture and analyze sports two hot technology research, mainly on the key technology of two-dimensional and three-dimensional sports movement tracking method reconstruction done in-depth research, and ultimately a feature-based tracking video Sports motion capture prototype system. It can be extracted from a fixed background of video clips in the body motion information, automatically generate motion capture data files, thus completing the sports capture process. Specifically system is the body's first label each joint position in the first frame, and then do feature-based tracking in subsequent frames to generate two-dimensional coordinates of the joint point sequence, and then make a three-dimensional reconstruction of the movement, and finally through the data conversion and re-organization, to form the desired motion capture data files. Experimental results show that this method has a bit of accuracy and practicality.

\section{Video analysis features tagging technology}

Based on the analysis of motion video processing, according to the degree of human intervention can be divided into two methods to identify and unmarked. Marked method refers to a subject joints affixed obvious markers, labels feature points, thereby tracking feature points. No identification method is not dependent on any marker or manual tagging, motion analysis and tracking information in accordance with the image sequence itself. Systematic application identification is very extensive, such systems can be divided according to the type of identification 
system active and passive systems [3]. Active systems using active identification, these identification are actually some of the glowing LED lights, each LED light signals of different frequencies or patterns in the control unit of the drive, and presents a high-contrast background, so this type of system for identification the track is very easy [4].

Feature-based motion analysis it is another common method, its main feature is the image from an image correspond to another image. Through the study of the human eye is similar to advanced visual system indicates that the human eye can be characterized by the space corresponding match in the considerable time interval to create the feeling of movement, which can track a visual target in long distance space. Feature-based methods generally involve two processes:

1) Feature selection. Image feature is due to the physical and geometrical characteristics of the scene so that grayscale image local region to produce significant changes in the formation. Therefore it means that there exists in the characteristics of the local area in the larger amount of information, but not in the image region feature should be only a small amount of information. Image feature extraction, which extract useful information and visual features from the image. Broadly speaking, the image is characterized by a clear image field separable, primitive characteristics or properties.

2) Feature matching. Before calculating the motion information, the need for the feature points on the object tracking frame by frame. Matching algorithms are introduced rigid constraints, have some technology, including template matching, matching structures, tree search to match, constraint relaxation matching and matching hypothesis testing. Feature matching is to establish a one to one relationship between the characteristics of the image sequence. Feature matching has been a very difficult problem.

\section{Human motion tracking method based on tagging feature}

The body's movement can be seen as a whole, but also can be decomposed into sub-parts of the Movement, the whole movement manifests itself in translation and rotation, while the sub-movement manifests itself at the site of a joint connection point within a certain range of freedom of movement. For example, the upper arm is connected to the shoulder point, we can only point to the shoulder joint axis within a certain range of motion, and cannot be separated shoulder. Rod model various parts of the body in a straight line represented by dots of each joint point of convergence. The main parameters of the rod-shaped model include the location of the body joints, bones connected to the joint between the bones of the length and direction, as shown in Figure 1 shows. Whereby the rod-shaped body model can describe different body posture. 2D bar-shaped model can be used as part of the calibration and describing two-dimensional human motion skeleton sequence of recovery after joint position, 3D rod model can be used to restore the body's various joints depth coordinate to be used for three-dimensional human skeleton sequence after the three-dimensional reconstruction description. The establishment of a hierarchical model kinesiology for monocular video sequence tracing the body. The body as a combination of multi-rigid, rigid connection between the respective joint, the model he established, the body's torso, limbs were used to fine stick representation [5-6]. This method reduces the computational complexity, improve the accuracy of the corresponding feature points, achieving the purpose of effective tracking human limb movement, having a certain value.

Walking human motion analysis of human movement has been important in the field of study. Because walking is, in general, and natural scene, the body of the most common forms of exercise. Therefore gait characteristics can be used as personal identification, and how to obtain, use gait biometrics research is an important issue. In this article, we dressed in black tights walk for the study of human movement, for monocular video walking human motion tracking, a feature introduced tracking algorithm. Video sequences attached MLD (Moving Light Displays) on the human body. MLD is a luminous markers, in this article for attachment points on each of the joints of the body, is used to mark the joint points, as shown in Figure 2.

In this article, the human movement as a connection type non-rigid motion, using the connection type non-rigid motion constraints that exist to guide the tracking process. Firstly, the structure of the 
constraint model, you can get the constraint relationship between the joints of the human body through the model. Then attached to the body for different parts of the characteristics of joints, according to the walking pattern of human movement in different parts of different sports, in order to take a different forecast track strategy to obtain the relevant characteristics of the detection window. Then in the corresponding window, by template matching method to detect feature, obtain the position of joints. Finally calculated motion parameters. The overall framework of the process of algorithm shown in Figure 3.

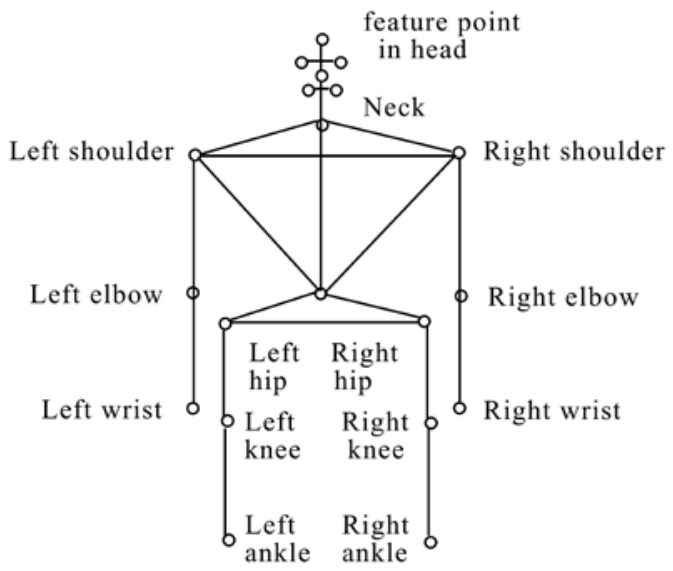

Figure 1. Feature labeling technique in sports video analysis
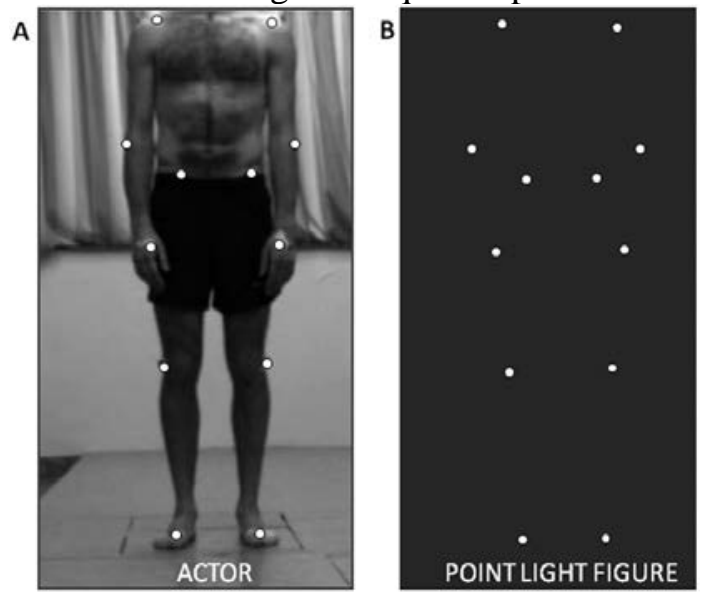

Figure 2. Video frames

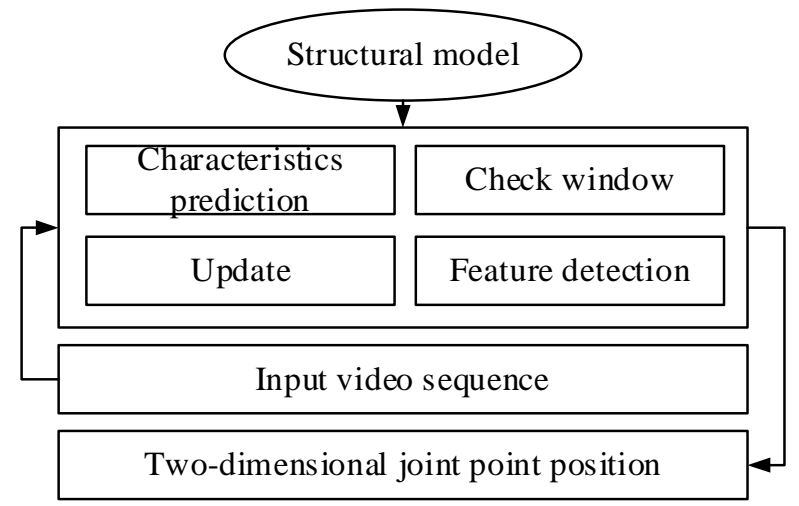

Figure 3. The overall framework of the process of algorithm

\section{Experiments}

We capture the performance of an actor with $n_{\text {cam }}$ synchronized and calibrated video cameras. The human body model comprises a kinematic skeleton and an attached body approximation modeled as a Sum of Gaussians. The skeleton consists of 58 joints, modeling a detailed spine and clavicles. Each joint is defined by an offset to its parent joint and a rotation represented in 
axis-angle form. The model features a total of 61 parameters, 58 rotational and an additional 3 translational. The skeleton features further a separate degree of freedom (DoF) hierarchy, consisting of $\mathrm{nDoF}$ pose parameters. For all the results in this paper we used a DoF hierarchy consisting of $n_{\text {DoF }}=43$ pose parameters. This construction allows the model to reproduce natural deformation of the spine, as a single DoF can model smooth bending. It also allows straight-forward creation of several different levels of detail without having to edit the kinematic joint hierarchy itself.

An outline of the processing pipeline is given in Figure 4. In a pre-processing step, we use a low number of manually segmented multi-view images showing example poses to estimate an actor specific body model. This model is then used for tracking the articulated motion of the actor from multi-view input videos. Each input image is converted into a SoG representation. Tracking starts with the estimated pose of the model in the previous frame, and optimizes the parameters such that the overlap similarity between the model and image SoG at the current frame is maximized.

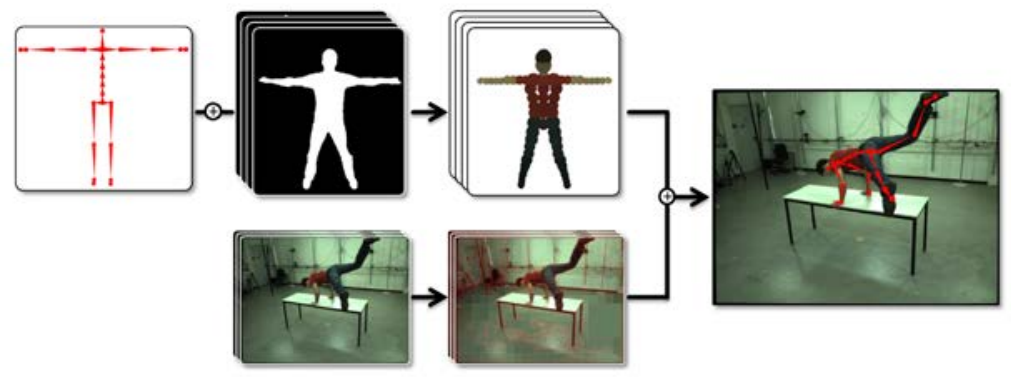

Figure 4. The processing pipeline

\section{Conclusion}

With the rapid expansion of video data, and how to find the required information has become an urgent problem. In this paper, for sports video, research-based video analysis and retrieval techniques. Its purpose is through the video content for computer processing, analysis and understanding of the structure and the establishment of an index to facilitate users to access, has important theoretical significance and application prospects. This paper mainly based video annotation retrieval and then edit a study, we propose a method to look forward to save video of blind search time and improve retrieval efficiency, and coaches in guiding the training under the guidance of empirical premise, better find more guidance policy from the retrieved video. In this paper, the early video after pretreatment, the upcoming collection label information into the video, on this basis, to complete the re-editing of the video. Logical structure and function of each sub-module of the system in this paper has done a detailed introduction, the final test results are given system. The system solves the search of video defects, and to be able to search for videos give intuitive synchronization circuit diagram display. Training for sports coaches save time blind search for videos, the more it reasonable guidance and decision-making provide a scientific basis.

\section{Reference}

[1] Siersdorfer S, San Pedro J, Sanderson M. Automatic video tagging using content redundancy[C]//Proceedings of the 32nd international ACM SIGIR conference on Research and development in information retrieval. ACM, 2009: 395-402.

[2] Karpathy A, Toderici G, Shetty S, et al. Large-scale video classification with convolutional neural networks[C]//Computer Vision and Pattern Recognition (CVPR), 2014 IEEE Conference on. IEEE, 2014: 1725-1732.

[3] Zhu G, Xu C, Huang Q, et al. Event tactic analysis based on broadcast sports video[J]. Multimedia, IEEE Transactions on, 2009, 11(1): 49-67.

[4] Soleymani M, Kierkels J J M, Chanel G, et al. A bayesian framework for video affective representation[C]//Affective Computing and Intelligent Interaction and Workshops, 2009. ACII 
2009. 3rd International Conference on. IEEE, 2009: 1-7.

[5] Filippova K, Hall K B. Improved video categorization from text metadata and user comments[C]//Proceedings of the 34th international ACM SIGIR conference on Research and development in Information Retrieval. ACM, 2011: 835-842.

[6] Legg P A, Chung D H S, Parry M L, et al. MatchPad: Interactive Glyph - Based Visualization for Real - Time Sports Performance Analysis[C]//Computer Graphics Forum. Blackwell Publishing Ltd, 2012, 31(3pt4): 1255-1264. 